\title{
Endothelial Cells Constituting Blood-nerve Barrier Have Highly Specialized Characteristics as Barrier-forming Cells
}

\author{
Yasuteru Sano ${ }^{1}$, Fumitaka Shimizu${ }^{1}$, Hiroto Nakayama1, Masaaki Abe ${ }^{1}$, Toshihiko Maeda ${ }^{1}$, \\ Sumio Ohtsuki ${ }^{2}$, Tetsuya Terasaki ${ }^{2}$, Masuo Obinata ${ }^{3}$, Masatsugu Ueda ${ }^{4}$, Ri-ichi Takahashi ${ }^{4}$ \\ and Takashi Kanda ${ }^{*}$ \\ ${ }^{1}$ Department of Neurology and Clinical Neuroscience, Yamaguchi University Graduate School of Medicine, \\ 1-1-1 Minami-kogushi, Ube, 755-8505, Japan, ${ }^{2}$ Department of Molecular Biopharmacy and Genetics, \\ Graduate School of Pharmaceutical Sciences, Tohoku University, Aoba, Aramaki, Aoba-ku, Sendai, \\ 980-8578, Japan, ${ }^{3}$ Department of Cell Biology, Institute of Development, Aging and Cancer, Tohoku University, \\ 4-1, Seiryomachi, Aoba-ku, Sendai, 980-8578, Japan, ${ }^{4}$ The YS Institute, Inc., 1198-4 Iwazo, Utsunomiya, \\ 321-0973, Japan
}

\begin{abstract}
In autoimmune disorders of the peripheral nervous system (PNS) such as Guillain-Barré syndrome and chronic inflammatory demyelinating polyradiculoneuropathy, breakdown of the blood-nerve barrier (BNB) has been considered as a key step in the disease process. Hence, it is important to know the cellular property of peripheral nerve microvascular endothelial cells (PnMECs) constituting the bulk of BNB. Although many in vitro models of the blood-brain barrier (BBB) have been established, very few in vitro BNB models have been reported so far. We isolated PnMECs from transgenic rats harboring the temperature-sensitive SV40 large T-antigen gene (tsA58 rat) and investigated the properties of these "barrier-forming cells". Isolated PnMECs (TR-BNBs) showed high transendothelial electrical resistance and expressed tight junction components and various types of influx as well as efflux transporters that have been reported to function at BBB. Furthermore, we confirmed the in vivo expression of various BBB-forming endothelial cell markers in the endoneurium of a rat sciatic nerve. These results suggest that PnMECs constituting the bulk of BNB have a highly specialized characteristic resembling the endothelial cells forming BBB.
\end{abstract}

Key words: blood-nerve barrier/peripheral nerve microvascular endothelial cells/tight junction/claudin-5/transporter

\section{Introduction}

The blood-nerve barrier (BNB) is one of the functional barriers sheltering the nervous system from circulating blood (Poduslo et al., 1994). BNB comprises the endoneurial microvasculature and the innermost layers of the perineurium. Tight junctions (TJs) between adjacent peripheral nerve

\footnotetext{
*To whom correspondence should be addressed: Takashi Kanda, Department of Neurology and Clinical Neuroscience, Yamaguchi University Graduate School of Medicine, 1-1-1 Minami-kogushi, Ube, Yamaguchi, 755-8505, Japan.

Tel: +81-83-622-2719, Fax: +81-83-622-2364

E-mail: tkanda@yamaguchi-u.ac.jp

Abbreviations: BSA, bovine serum albumin; SV40, simian virus 40; PBS, phosphate-buffered saline; EC, endothelial cell; CNS, central nervous system; PNS, peripheral nervous system; tsA58 rat, transgenic rat harboring temperature-sensitive SV40 large T-antigen gene; TR-BBB, conditionally immortalized rat brain capillary endothelial cell line; TR-BNB, conditionally immortalized rat peripheral nerve microvascular endothelial cell line.
}

microvascular endothelial cells (PnMECs) and between perineurial cells, in addition to the lack of vesicular transport, are responsible for BNB function (Bell et al., 1984; Latker et al., 1991). Blood-borne substances can reach the endoneurial extracellular space by crossing either the endoneurial vascular endothelium or the perineurium. Many studies (Soderfeldt, 1974; Boddingius, 1984; Weerasuriya and Rapoport, 1986; Weerasuriya et al., 1989) indicated that perineurial permeability is much lower than that of endoneurial microvessels against various substances. Hence, PnMECs constituting the bulk of microvessels in the endoneurium can be considered the real interface between blood and peripheral nerves. Recently, breakdown of BNB has been considered as an initial key step in many autoimmune disorders of the peripheral nervous system including Guillain-Barré syndrome, chronic inflammatory demyelinating polyradiculoneuropathy (CIDP), and paraprotemic neuropathy (Lach et al., 1993; Kanda et al., 1994, 2000, 
2004). Because PnMECs are the structural basis of BNB, as indicated above, investigation of the cellular characteristics of PnMECs using cell culture technique may provide new insights into the pathogenesis of immune-mediated neuropathies. To date, brain microvascular endothelial cells (BMECs) have been successfully isolated in many laboratories and utilized for studying the molecular and cellular basis of BBB (Terasaki et al., 2003). On the other hand, only a few reports concerning the successful culture of PnMECs have been available thus far (Argall et al., 1994; Kanda et al., 1997; Iwasaki et al., 1999). This is probably due to the technical difficulty in isolating PnMECs from a peripheral nerve. Although many endothelial cells isolated from humans and animals need to be introduced immortalizing genes, such as the SV 40 large T-antigen, to gain abilities for stable proliferations, these immortalized cells often end up loosing their natural properties in vivo (Terasaki et al., 2003). Recently, transgenic rats harboring temperaturesensitive simian virus 40 large T-antigen (tsA58) gene have been developed as a source of conditionally immortalized cell lines (Takahashi et al., 1999). Cultured cells derived from this animal can be easily immortalized by activating the tsA58 gene at a permissive temperature of $33^{\circ} \mathrm{C}$ and can have in vivo functions at a nonpermissive temperature of $37^{\circ} \mathrm{C}$ or $39^{\circ} \mathrm{C}$ (Takahashi et al., 1999; Hosoya et al., 2000). Here, we successfully isolated PnMECs from the tsA58 rats and investigated the properties of these cells. In addition, we examined whether in vivo microvessels in the endoneurium express barrier-related molecules, which have been reported to function at $\mathrm{BBB}$. This study should establish a new paradigm for understanding the nature of PnMECs forming the bulk of BNB.

\section{Materials and Methods}

\begin{abstract}
Animals
The origin and characteristics of the transgenic rats harboring the temperature sensitive SV40 large T antigen gene have been previously described (Takahashi et al., 1999). Wistar rats utilized for immunohistochemistry and reverse transcription-polymerase chain reaction (RT-PCR) analysis were purchased from Charles River Laboratories Inc. (Yokohama, Japan). All investigations using rats described in this report conform to the requirements established by the Institute of Laboratory Animals, Yamaguchi University Graduate School of Medicine.
\end{abstract}

\section{Culture media}

Dissecting medium (DM) contained Dulbecco's modified Eagle's medium (DMEM) (Sigma, St. Louis, MO, U.S.A.) supplemented with 5\% fetal bovine serum (FBS) (Sigma), $100 \mathrm{U} / \mathrm{ml}$ penicillin (Sigma), $100 \mu \mathrm{l} / \mathrm{ml}$ streptomycin (Sigma), and $25 \mathrm{ng} / \mathrm{ml}$ amphotericin B (Invitrogen, Grand Island, NY, U.S.A.). Endothelial cell
(EC) growth medium contained EBM-2 medium (Cambrex, Walkersville, MD, U.S.A.) supplemented with 20\% FBS, EGM-2 MV (Cambrex), $100 \mathrm{U} / \mathrm{ml}$ penicillin (Sigma), $100 \mu \mathrm{l} / \mathrm{ml}$ streptomycin (Sigma), and $25 \mathrm{ng} / \mathrm{ml}$ amphotericin B (Invitrogen).

\section{Isolation of ts $A 58$ rat PnMECs forming BNB and endothelium from ts $A 58$ rat aorta}

PnMECs were isolated in accordance with a previously described procedure (Kanda et al., 1997) with modification. Briefly, the sciatic nerve was removed from a rat killed by euthanasia procedures. The epi- and perineuria were carefully stripped off with fine forceps, mimicking the teased fiber preparation for the peripheral nerve pathology. Next, the endoneurium was finely minced with a razor blade and digested with $0.25 \%$ collagenase type I (Sigma) in $1 \times$ Hank's balanced salt solution (HBSS) (Invitrogen) at $37^{\circ} \mathrm{C}$ for 2 hr. After centrifugation, the pellet was washed and placed onto a type I collagen-coated dish. Cells were cultured at $37^{\circ} \mathrm{C}$ during the first 48-72 hr to allow the cells to attach themselves to the dish. Cells were subsequently cultured at $33^{\circ} \mathrm{C}$ in a humidified atmosphere of $5 \% \mathrm{CO}_{2}$ and $95 \%$ air. When the EC colonies grew sufficiently for cloning, they were picked up with a cloning cup. As ECs grew, non-ECs such as pericytes, fibroblasts, and Schwann cells also appeared and gradually began to occupy the culture area of the dish. These non-ECs were scratched and removed mechanically with a sterilized pointed rubber. After that, ECs were isolated and cultured for $2-3$ weeks at $33^{\circ} \mathrm{C}$. These PnMECs (TR-BNBs) showed immortality at a permissive temperature of $33^{\circ} \mathrm{C}$. ECs from ts A58 rat aorta were isolated in accordance with a previously reported method (Kobayashi et al., 2005).

\section{Identification of PnMECs}

PnMECs were identified by the following three criteria: spindlefiber-shaped morphology; immunoreactivity to an anti-von Willebrand factor antigen antibody, and uptake of 1,1'-dioctacecyl$3,3,3 ', 3$ ', tetramethyl indocarbocyanine perchlorate acetylated lowdensity lipoprotein (DiI-Ac-LDL) (Biogenesis, Poole, England). To label with DiI-Ac-LDL, cells were incubated with $10 \mu \mathrm{g} / \mathrm{ml}$ DiI-Ac-LDL at $33^{\circ} \mathrm{C}$ in a culture medium overnight. Cells were subsequently viewed under a fluorescence microscope (Olympus, Tokyo, Japan). PnMECs incorporated bright DiI-Ac-LDL particles into their cytoplasm.

\section{Immunocytochemistry}

Cells $\left(1 \times 10^{6}\right)$ were cultured on a rat tail collagen type I-coated 30$\mathrm{mm}$ dish (Becton Dickinson) at $33^{\circ} \mathrm{C}$ until reaching confluence (24-48 hr). After an additional incubation at a nonpermissive temperature of $37^{\circ} \mathrm{C}$ for $24 \mathrm{hr}$, cells were washed three times with PBS. For von Willebrand factor immunocytochemistry, cells were fixed in 4\% paraformaldehyde (Wako, Osaka, Japan) for $15 \mathrm{~min}$ at room temperature. Next, cells were permeabilized with $0.1 \%$ Triton X-100 (Sigma) for 10 min and then blocked with 1\% BSA in PBS for $1 \mathrm{hr}$. After several washes with PBS, cells were incubated 
with the mouse anti-human von Willbrand factor antibody (1:100 dilution) (Dako, A/S, Denmark) with blocking solution at $4{ }^{\circ} \mathrm{C}$ overnight. Cells were subsequently washed with PBS and then incubated with FITC-conjugated anti-mouse IgG (1:100 dilution) (Zymed, CA, U.S.A.) for $1 \mathrm{hr}$ at room temperature. For claudin-5 immunocytochemistry, cells were fixed in $100 \%$ ethanol for 30 $\min$ at $4{ }^{\circ} \mathrm{C}$. After treatment with $1 \%$ Triton X-100/PBS for $10 \mathrm{~min}$ at room temperature, the cells were blocked with $1 \%$ BSA/PBS for $1 \mathrm{hr}$. After several washes with PBS, cells were incubated with a rabbit anti-mouse claudin-5 antibody (1:100 dilution) (Zymed, CA, U.S.A.) with a blocking solution at $4^{\circ} \mathrm{C}$ overnight. Cells were subsequently washed with PBS and then incubated with FITCconjugated anti-rabbit IgG (1:100 dilution) (Zymed, CA, U.S.A.) for $1 \mathrm{hr}$ at room temperature. Fluorescence was detected with a fluorescence microscope (Olympus).

\section{Transendothelial electrical resistance (TEER) study}

Transendothelial electrical resistance (TEER) reflects the barrier properties of the endothelium (Deli et al., 2005). The transwell inserts (pore size, $0.4 \mu \mathrm{m}$; effective growth area, $0.3 \mathrm{~cm}^{2}$; BD Bioscience, New Jersey, U.S.A.) were coated with rat tail collagen type-I (BD Bioscience) in accordance with the manufacturer's instruction. At $1.0 \times 10^{6}$ cells/insert, cells were seeded on these collagen-coated culture inserts at the nonpermissive temperature $\left(37^{\circ} \mathrm{C}\right)$ (Hosoya et al. 2000$)$. After attaching themselves with confluence to the bottom of the insert (24-36 hr), the TEER of cell layers was measured with a Millicell electrical resistance apparatus (Endohm-6 and EVOM, World Precision Instruments, Sarasota, FL, U.S.A.). TR-BBB13 cells, derived from the tsA58 rat brain, which have been thought to suppress claudin-5 expression (Hosoya et al., 2000; Ohtsuki et al., 2007) and human umbilical vein endothelial cells (HUVECs) (Japan Health Sciences Foundation, Osaka, Japan) were used as control endothelial cells without barrier properties. Statistical significance was evaluated using Student's t-test.

\section{RT-PCR analysis}

After an incubation at the nonpermissive temperature of $37^{\circ} \mathrm{C}$ for $24 \mathrm{hr}$, cells were washed three times with PBS. Total RNA was prepared from PBS-washed cells or the rat cerebrum using an RNeasy ${ }^{\circledR}$ Plus Mini kit (Qiagen, Hilden, Germany). RT and PCR amplification were carried out with TAKARA PCR Thermal Cycler Dice (TakaRa, Otsu, Japan). Single-stranded cDNA was synthesized from $50 \mathrm{ng}$ of total RNA using the StrataScript ${ }^{\circledR}$ First Strand Synthesis System (STRATAGENE ${ }^{\circledR}$, Cedar Greek, TX, U.S.A.) with an oligo-dT primer, and sequential PCR was performed with TaKaRa Ex $\mathrm{Taq}^{\circledR}(\mathrm{TaKaRa})$. Temperature cycling conditions for each primer consisted of $5 \mathrm{~min}$ at $94^{\circ} \mathrm{C}$ followed by $35-40$ cycles for $1 \mathrm{~min}$ at $94^{\circ} \mathrm{C}, 1 \mathrm{~min}$ at $55-65^{\circ} \mathrm{C}$, and $1 \mathrm{~min}$ at $72^{\circ} \mathrm{C}$, with a final extension for $10 \mathrm{~min}$ at $72^{\circ} \mathrm{C}$. The sequence specificity of each rat primer pair and its reference are shown in Table I. The PCR products were visualized by ethidium bromide staining following resolution on $2 \%$ agarose gel. Products were compared with a 50-bp ladder (Fermentas, Burlington, Canada) to estimate band size. The size of each amplified product corresponded to the expected size described in each literature we had referred. For the RT-PCR analysis of rat tissues, the cerebrum, sciatic nerve, aorta, and kidneys were removed from a nontransgenic Wistar rat killed by euthanasia procedures. The cerebral cortex, endoneurium of a sciatic nerve, aorta, and kidneys were separately minced and homogenized for total RNA extraction. After singlestranded cDNA was synthesized from $400 \mathrm{ng}$ of total RNA derived from each tissue, PCR and sequential examinations were performed using the same procedure described above.

\section{Immunohistochemistry}

Rabbit polyclonal antibodies against human claudin-12, human occludin and mouse claudin-5 were purchased from Zymed (San Francisco, CA, U.S.A.). A rabbit polyclonal antibody against human GLUT-1 was purchased from Santa Cruz Biotechnology (CA, U.S.A.). A mouse monoclonal antibody against human pglycoprotein (p-gp) was purchased from Signet Laboratories (Dedham, MA, U.S.A.). The brain and sciatic nerve were removed from a Wistar rat killed by the euthanasia procedures. Tissues were snap-frozen by immersion into isopentane/liquid nitrogen. Cryostat sections $(10 \mu \mathrm{m})$ were mounted on poly-L-lysine-coated slides and air-dried. After a 15 -min fixation with $4 \%$ paraformaldehyde at $4{ }^{\circ} \mathrm{C}$, they were exposed to $0.3 \% \mathrm{H}_{2} \mathrm{O}_{2} /$ methanol for 10 min at room temperature. Sections were then preincubated in PBS supplemented with $10 \%$ normal goat serum for $1 \mathrm{hr}$ before incubating overnight with the primary antibody diluted in PBS at $4{ }^{\circ} \mathrm{C}$. The anti-occludin antibody was used at 1/800 dilution, whereas others were used at 1/400 dilution. Sections were then rinsed with PBS three times before incubation for $1 \mathrm{hr}$ with peroxidaseconjugated secondary antibodies (Nichirei, Tokyo, Japan) at room temperature. The reaction product indicating immunoreactivity in sections was developed with diaminobenzidine (Nichrei).

\section{Results}

\section{Establishment of TR-BNBS}

We had successfully isolated PnMECs from sciatic nerves of a tsA58 rat. TR-BNBs were closely packed and showed a spindle-fiber-shaped morphology that has been well recognized to be ECs constituting the barrier system (Kanda et al., 1997; Hosoya et al., 2000) (Fig. 1A). In contrast, ECs of the tsA58 rat aorta, as representative cells that do not form the blood-tissue barrier, showed a "cobblestone-like appearance", not a spindle-fiber-shaped morphology (Fig. 1B). Almost $100 \%$ of the cells were positive for DiI-Ac-LDL, indicating excellent purity (Fig. 1C, D). The endothelial origin of these cells was also supported by the detections of the factor VIII/vWF antigen by RT-PCR analysis (Fig. 1E) and immunocytochemistry (Fig. 1F). 
A

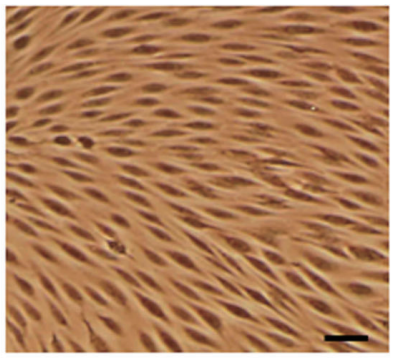

$\mathbf{B}$
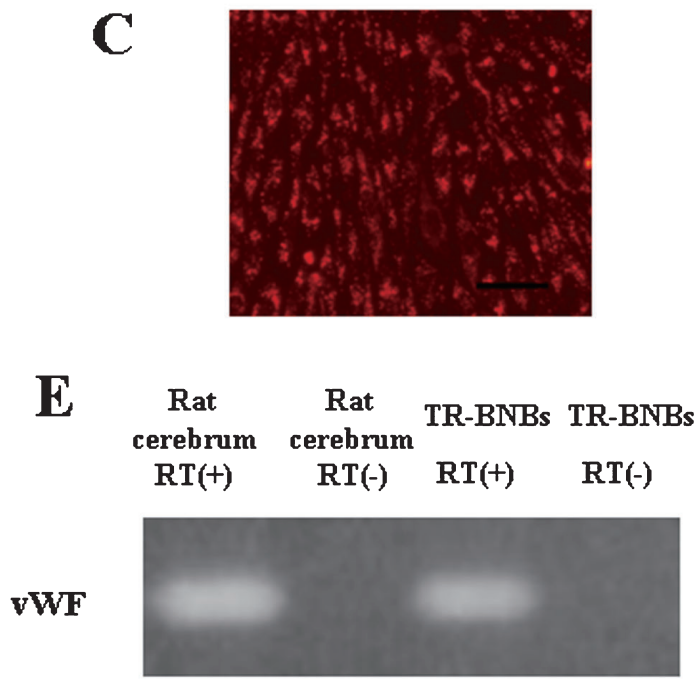

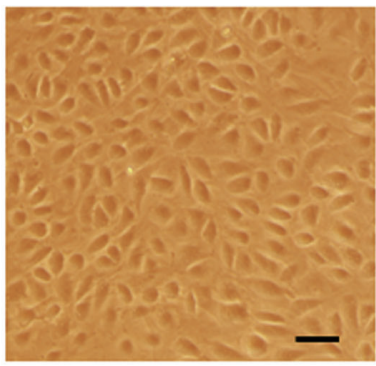

$\mathbf{D}$

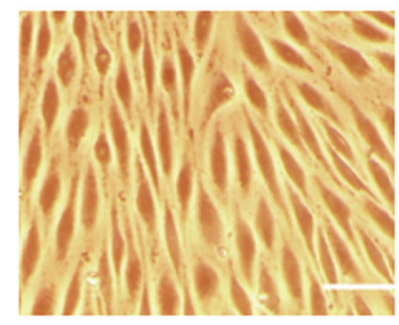

$\mathbf{F}$

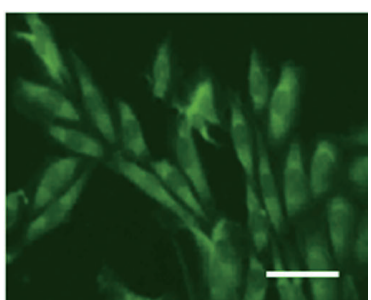

Fig. 1. Spindle-fiber-shaped morphology and endothelial properties of isolated TR-BNBs. (A): Phase contrast micrograph of TR-BNBs cultured at $33^{\circ} \mathrm{C}$. (B): Cobblestone-like appearance of tsA58 rat aortic endothelial cells. (C) and (D): Fluorescence micrograph of TR-BNBs with DiI-Ac-LDL (C) and phase contrast micrograph of the same field (D). (E): Expression of von Willebrand factor gene in TR-BNBs detected by RT-PCR analysis. F: Immunostaining of TR-BNBs using anti-von Willebrand factor antibody. Scale bars, $100 \mu \mathrm{m}$.

\section{TR-BNBs expressing claudin-5 have high TEER}

The TEER of TR-BNBs was significantly higher than that of HUVECs (Fig. 2A). Furthermore, the TEER of TRBNBs was also higher than that of TR-BBBs (TR-BBB13) (Fig. 2A), which have been reported to suppress claudin-5 expression (Ohtsuki et al., 2007). In addition, we investigated whether the TR-BNBs express claudin-5. Claudin5 was localized at the cell-cell boundaries in TR-BNBs (Fig. 2B).

\section{Expression of tight junction molecules in TR-BNBs}

To investigate the mRNA expression of components of tight junctions in PnMECs, total RNA was isolated from TRBNBs and processed for RT-PCR analysis. The expressions of occludin, claudin-5, claudin-12, junctional adhesion molecule 1 (JAM1), zonula occludens 1 (ZO-1), and zonula occludens 2 (ZO-2), corresponding to tight junction components of ECs forming BBB (Lai et al., 2005) were confirmed in TR-BNBs (Fig. 3). The sizes of amplified fragments of each molecule were identical to previously reported values (Table I).

\section{Expression of influx transporters in TR-BNBs}

The mRNA expression of various blood-to-brain influx transporters in TR-BNBs was examined by RT-PCR analysis using a specific primer set for each rat transporter (Table I). Glucose transporter 1 (GLUT1), which takes in Dglucose from circulating blood, was expressed in TR-BNBs (Fig. 4). The expression of a large amino acid transporter (system L), which consists of large neutral amino acid transporter-1 (LAT-1) and 4F2hc, was examined. LAT-1 and 4F2hc mRNAs were amplified at their expected sizes (Fig. 4). Creatine plays a pivotal role in the storage of phosphatebound energy in the brain, and creatine transporter (CRT) supplies creatine to the brain via BBB. (Ohtsuki et al., 2002). CRT was also expressed in TR-BNBs (Fig. 4). On the other hand, monocarboxylic acid transporter 1 (MCT1), which has been reported to work as a blood-to-brain transporter of monocarboxylic acids such as lactate (Kido et al., 
$\mathbf{A}$

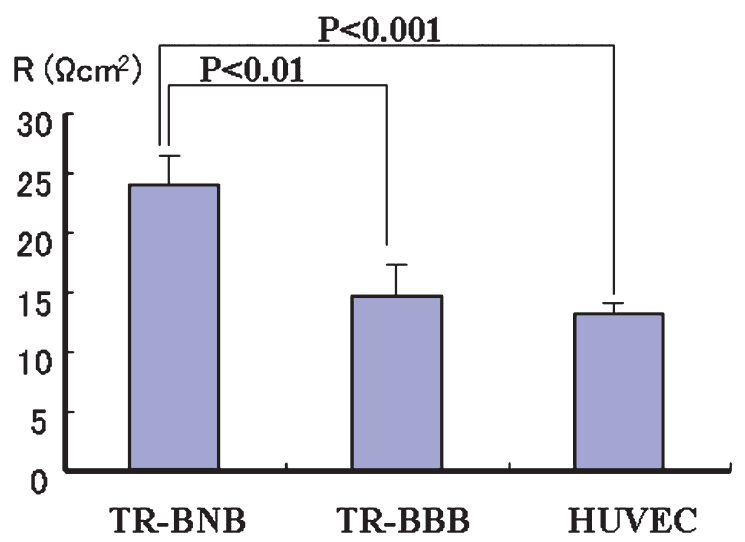

B

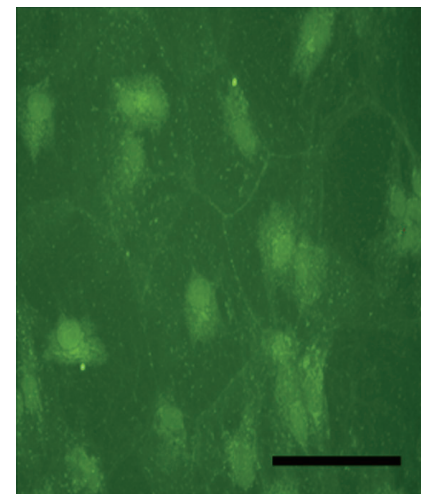

Fig. 2. TEER and claudin-5 expression in TR-BNBs. (A) Transendothelial electrical resistance (TEER) values of TR-BNBs, TR-BBBs, and HUVECs. TEER values recorded in ohms $(\Omega) \times$ insert area were corrected to $\Omega \mathrm{cm}^{2}$. TEER values of inserts with medium alone were subtracted from TEER values of inserts with confluent monolayers of each cell line. (mean $\pm \mathrm{SD}, \mathrm{n}=3$ in each experiment). (B) Expression and localization of claudin-5 in TR-BNBs. Claudin-5 was detected at the cell-cell boundaries of TR-BNBs. Scale bars, $100 \mu \mathrm{m}$.

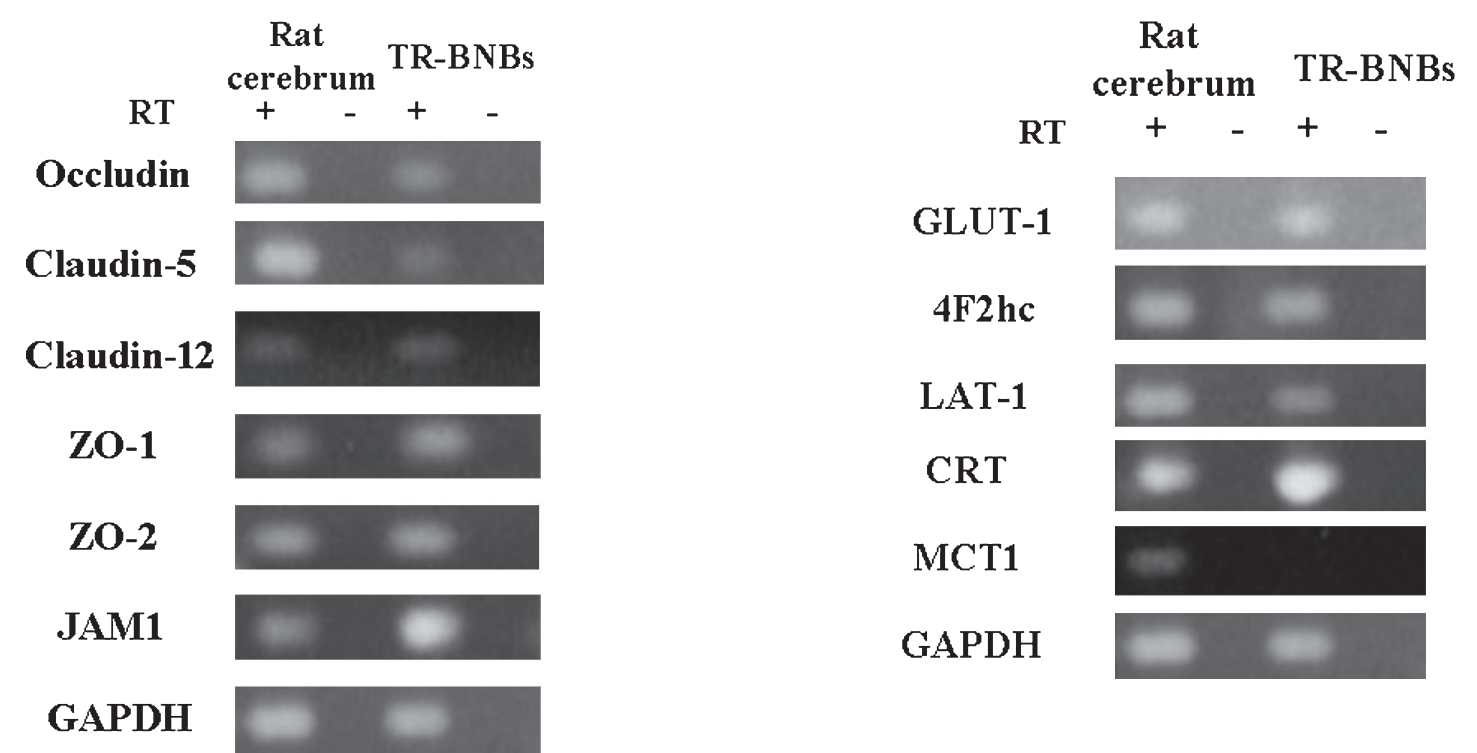

Fig. 3. mRNA expression of TJ molecules in TR-BNBs. Expression of occludin, claudin-5, claudin-12, ZO-1, ZO-2, and JAM1 mRNAs in the rat cerebrum and TR-BNBs was investigated by RT-PCR analysis with a specific primer set for each gene. Reactions were performed against total RNA with (+) or without $(-)$ reverse transcription. The amplified products for all molecules were detected in TR-BNBs.

2000), was not expressed in TR-BNBs (Fig. 4).

\section{Expression of brain-to-blood efflux transporters in TR- BNBS}

To elucidate whether PnMECs at BNB, similarly to BMECs at $\mathrm{BBB}$, carry out active efflux transport of various drugs

Fig. 4. mRNA expression of influx transporters in TR-BNBs. Expression of GLUT1, LAT-1, 4F2hc, MCT1, and CRT mRNAs in the rat cerebrum and TR-BNBs was investigated by RT-PCR analysis with a specific primer set for each gene. Reactions were performed against total RNA with $(+)$ or without $(-)$ reverse transcription. The amplified products for all molecules but MCT1 were detected in TR-BNBs.

and unnecessary metabolites for neurons from the peripheral nervous parenchyma to the inner lumen of a capillary, we investigated mRNA expression of diverse brain-to-blood efflux transporters that have been reported to function at BBB. Multidrug resistance gene 1a (Mdr1a), multidrug resistance associated protein 1 (Mrp1), and ATP-binding cassette subfamily G member 2 (ABCG2), which mediate 
Table I. RAT PRIMER PAIRS UTILIZED IN RT-PCR ANALYSES

\begin{tabular}{|c|c|c|c|}
\hline molecule & sense & antisense & reference \\
\hline Occludin & 5'-GCCTTTTGCTTCATCGCTTCC-3' & 5'-AACAAGATTAAAGCAAAAGCCAC-3' & Hosoya et al., 2000 \\
\hline Claudin-5 & 5'-GCAGAGTACAGGGCACATGC-3' & 5'-TAGTTCTTCTTGTCGTAATCGCC-3' & Hosoya et al., 2000 \\
\hline Claudin-12 & 5'-GCGGCCGCGACAGGCTGTTTGGAGAA-3' & 5'-GCGGCCGCCACTGATGGGTAAGGAACA-3' & Ohtsuki et al., 2007 \\
\hline JAM1 & 5'-ACAGCCATGAGGTCAGAGGCT-3' & 5'-ACCTAGAAGACATTGAAGGCATC-3' & Tomi and Hosoya, 2004 \\
\hline ZO-1 & 5'-GCGAGGCATCGTTCCTAATAAG-3' & 5'-TCGCCACCTGCTGTCTTTG-3' & Shi and Zheng, 2005 \\
\hline $\mathrm{ZO}-2$ & 5'-TCACAGCTGCAGGTGTCACA-3' & 5'-TGCTGTTCTGGGTTGAGGAACT-3' & Shi and Zheng, 2005 \\
\hline GLUT-1 & 5'-GATGATGAACCTGTTGGCCT-3' & 5'-AGCGGAACAGCTCCAAGATG-3' & Tomi and Hosoya, 2004 \\
\hline $4 \mathrm{~F} 2 \mathrm{hc}$ & 5'-CTCCCAGGAAGATTTTAAAGACCTTCT-3' & 5'-TTCATTTTGGTGGCTACAATGTCAG-3' & Hosoya et al., 2000 \\
\hline LAT-1 & 5'-CAAGCTCTGGATCGAGCTGCTC-3' & 5'-TGTGGGTGGATCATGGAGAGG-3' & Hosoya et al., 2000 \\
\hline MCT1 & 5'-GAAAAACTCAAGTCCAAAGAGTCT-3' & 5'-AGAAGCCCAAGAAGACAATGAAA-3' & Hosoya et al., 2001 \\
\hline CRT & 5'-GAAATGGTGCTGGTCCTTCTTCAC-3' & 5'-GTCACATGACACTCTCCACCACGA-3' & Tomi and Hosoya, 2004 \\
\hline Mdr1a & 5'-ACAGAAACAGAGGATCGC-3' & 5'-CGTCTTGATCATGTGGCC-3' & Hosoya et al., 2000 \\
\hline Mrp1 & 5'-CTGGCTTGGTGTGAACTGAT-3' & 5'-AGGCTCTGGCTTGGCTCTAT-3' & Hosoya et al., 2000 \\
\hline ABCG2 & 5'-CAATGGGATCATGAAACCTG-3' & 5'-GAGGCTGATGAATGGAGAA-3' & Hosoya et al., 2004 \\
\hline OAT3 & 5'-ATCTCATCAACATCTATTGGCT-3' & 5'-CAGAGAGAGACAGAAGGTCA-3' & Ohtsuki et al., 2005 \\
\hline Oatp2 & 5'-GAGTACCTTCTGTCTTTCCTTAGCTA-3' & 5'-AACTAACGCAATCTGGCTTAACCAAG-3' & Ito et al., 2003 \\
\hline vWF & 5'-GCCTCTACCAGTGAGGTTTTGAAG-3' & 5'-АТСТCATCTCTTCTCTTCTGCTCCAGC-3' & Hosoya et al., 2000 \\
\hline GAPDH & 5'-TGATGACATCAAGAAGGTGGTGAAG-3' & 5'-TCCTTGGAGGCCATGTAGGCCAT-3' & Ohtsuki et al., 2005 \\
\hline
\end{tabular}

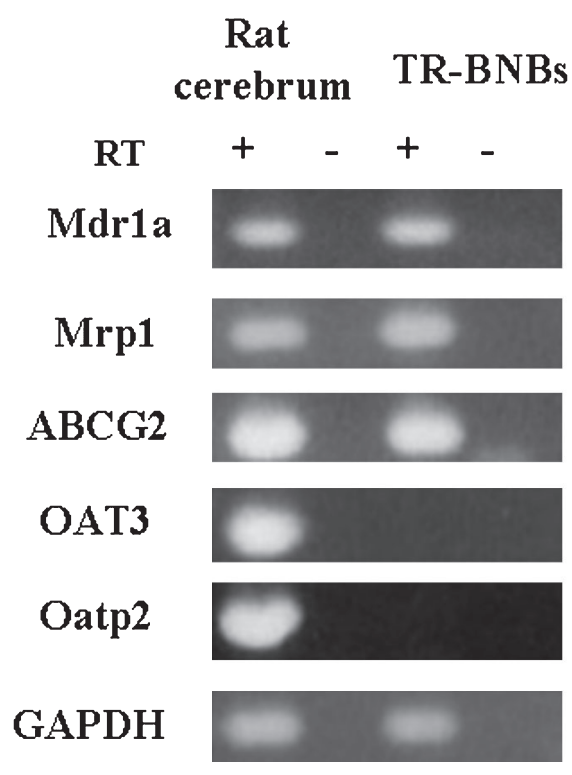

Fig. 5. mRNA expression of brain-to-blood efflux transporters in TRBNBs. Expression of Mdr1a, Mrp1, ABCG2, OAT3, and oatp2 mRNAs in the rat cerebrum and TR-BNBs was investigated by RT-PCR analysis with a specific primer set for each gene. Reactions were performed against total RNA with $(+)$ or without $(-)$ reverse transcription. Although the amplified products for Mdr1a, Mrp1, and ABCG2 were detected in TR-BNBs, those for OAT3 and oatp2 were not detected.

brain-to-blood efflux transport at BBB, were all detected at the mRNA level in TR-BNBs (Fig. 5). However, organic anion transporter 3 (OAT3) and oatp2, which are other types of brain-to-blood efflux transporter, were not detected

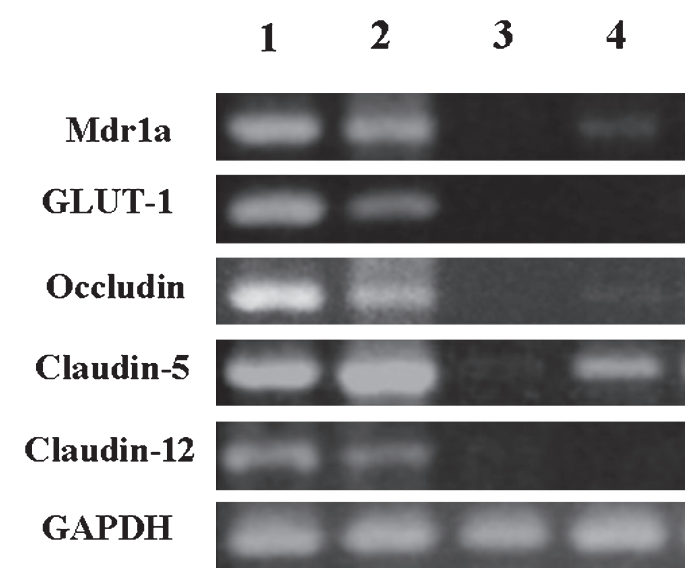

Fig. 6. mRNA expression of barrier-related molecules in various organs from a nontransgenic Wistar rat. Expression of Mdr1a, GLUT-1, occludin, claudin-5, and claudin-12 in rat tissues was investigated with primer sets listed in Table I. All molecules examined were detected in the cerebrum (lane 1) and sciatic nerve (lane 2). These molecules were not detected in the aorta (lane 3). Only Mdrla, occludin, and claudin-5 were detected in the kidneys (lane 4).

\section{in TR-BNBs (Fig. 5).}

\section{In vivo detection of $B B B$-forming $E C$ markers in the endoneurium of a rat sciatic nerve}

To verify the results of in vitro analysis, we examined the expression of tight junction molecules and transporters in vivo. RT-PCR experiments indicated that the cerebrum and 

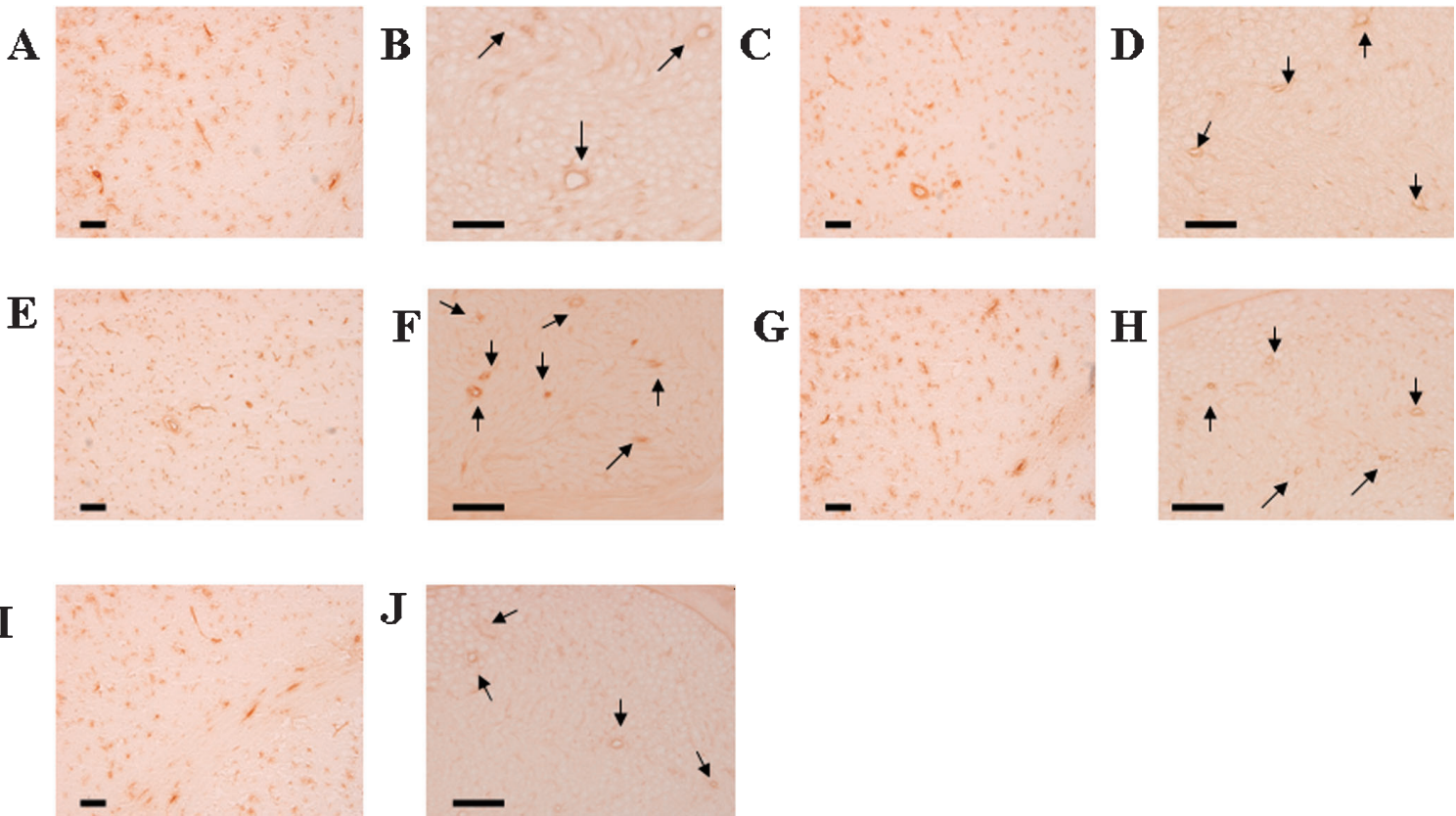

Fig. 7. Immunohistochemical staining of tissue sections of the rat cerebrum (A, C, E, G and I) and sciatic nerve (B, D, F, H and J). Endoneurial microvessels (arrows) in the rat sciatic nerve as well as microvessels in the rat cerebrum showed positive staining for p-glycoprotein (A and B), GLUT-1 (C and D), claudin-5 (E and F), occludin (G and H), and claudin-12 (I and J). Scale bars, $100 \mu \mathrm{m}$.

endoneurium of a sciatic nerve express the mRNAs of Mdr1a, GLUT-1, occludin, claudin-5, and claudin-12 (Fig. $6)$. On the other hand, the aorta expressed none of the molecules and the kidneys expressed only the mRNAs of Mdr1a, occludin, and claudin-5 (Fig. 6). These results indirectly provided the possibility that only ECs in the brain and endoneurium have common fundamental barrier properties and those of other tissues and organs, such as the aorta and kidneys do not. Microvessels in the cerebrum and endoneurium in the sciatic nerve expressed p-glycoprotein, GLUT-1, claudin-5, occludin, and claudin-12 (Fig. 7), corresponding to the detection of the mRNAs of these barrier-related proteins in RT-PCR experiments.

\section{Discussion}

Cerebral microvascular endothelial cells forming BBB have TJs that are critical for maintaining low permeability. To date, several proteins have been reported to be localized at TJs including occludin (Furuse et al., 1993), claudin-5 (Nitta et al., 2003), claudin-12 (Nitta et al., 2003; Ohtsuki et al., 2007), ZO-1, ZO-2 (Biernacki et al., 2004), and JAM (Bazzoni et al., 2000). Among these proteins, occludin, claudin-5 and ZO-1 have been detected in PnMECs in human sural nerve specimens by immunohistochemistry (Kanda et al., 2004). Hence, it can be expected that these TJ molecules might also play important roles at the cell-cell boundaries of PnMECs in BNB. Ohtsuki et al. reported that the exogenous expression of claudin-5 induces barrier properties in cultured rat brain capillary ECs (Ohtsuki et al., 2007). On the other hand, Nitta et al. reported the sizeselective opening of BBB in claudin-5-deficient mice (Nitta et al., 2003). In these mice, TJs with a normal appearance are present at the brain capillary endothelial cell-cell contact regions, but leakage of small molecules $(<800 \mathrm{Da})$, although not larger molecules, is observed at BBB. Furthermore, Kanda et al. reported that the percentage of claudin5-positive microvessels in the endoneurium of patients suffering from CIDP was significantly decreased compared with that of patients having noninflammatory neuropathies (Kanda et al., 2004). In the present study, we demonstrated the expressions of TJ components that are common between BMECs and PnMECs. In addition, our results indicated that TR-BNBs, which express claudin-5 at cell-cell boundaries, have significantly higher TEER than HUVECs as well as TR-BBBs, which do not express claudin-5 (Ohtsuki et al., 2007). Interestingly, Honda et al. reported that adrenomedullin increased the TEER of rat BMEC monolayer through the expression of claudin-5 (Honda et al., 2006). These data and our results indicate that claudin- 5 might be a key molecule in maintaining the integrity of TJs in BNB as well as BBB, and malfunction of this molecule might lead to the breakdown of BNB in many autoimmune peripheral neuropathies.

We would like to determine whether various transporters 
functioning at BBB are also present in PnMECs. Although some transporters, for example GLUT1 and p-gp, have been determined to be present on microvessels in the endoneurium by immunohistochemical analyses (Kanda et al., 1997; Saito et al., 1997), no studies have shown the mRNA expression of influx and efflux transporters in PnMECs forming the bulk of BNB. Therefore, we investigated the expression of various influx and efflux transporters in TRBNBs by RT-PCR analysis. We showed that TR-BNBs expressed GLUT-1, LAT1, and 4Fhc. Rechthand et al. (1985) disclosed the facilitated transport of D-glucose from blood into peripheral nerves. Wadhwani et al. (1990) demonstrated the facilitated transport of L-phenylalanine across the BNB of rat sciatic nerves. L-phenylalanine is considered to be one of the substrates of system $\mathrm{L}$, which is composed of a heterodimer of the 4F2hc heavy chain and the LAT1 light chain (Kanai et al., 1998). Hence, D-glucose and Lphenylalanine might be preferentially transported from circulating blood into the peripheral nervous parenchyma via GLUT1 and system L in BNB. Recently, creatine has been determined as a major energy-storing source of neuron in the brain and CRT has been reported to play an important role in the uptake of creatine from blood (Ohtsuki et al., 2002). Although there is no direct evidence that peripheral nerves use creatine as an energy-storing source, our results suggest that peripheral nerves also use creatine incorporated via CRT. MCT1 has been reported to be expressed at rat $\mathrm{BBB}$, and supply lactate as an energy source to the brain (Kido et al., 2000). Interestingly, we were not able to detect MCT1 mRNA in TR-BNBs; this indicates that peripheral nerves do not consume lactate as energy even during periods of starvation.

BBB is also involved in the brain-to-blood transport of many drugs and endogenous organic anions via efflux transporters. ABC transporters, such as Mdrla (p-gp) and Mrp1, mediate brain-to-blood efflux transport coupled with hydrolysis and operate to limit drug permeability to the brain (Terasaki et al., 2003; Pardridge, 2005). It is also well established that ABCG2 functions as a high-capacity efflux transporter of multiple drugs, such as mitoxantrone and doxorubicin, at BBB (Jonker et al., 2005). We demonstrated the mRNA expressions of Mdr1a, Mrp1, and ABCG2 in PnMECs. These results indicate that the nerve fibers of the peripheral nervous system might also be protected against harmful drugs by these efflux transporters. On the other hand, we were not able to detect OAT3 and oatp2 mRNAs in TR-BNBs. Homovanillic acid (HVA) is a major metabolite of dopamine and has been considered to be excreted from the brain to blood by OAT3 (Mori et al., 2003). Dehydroepiandrosterone (DHEAS), a neurosteroid that can interact with GABA type A receptors and sigma receptors, increases memory and learning ability and protects neurons against excitatory amino-acid-induced neurotoxicity (Asaba et al., 2000). Oatp2 has been reported to be involved in the brain-to-blood transport of DHEAS. The absence of OAT3 and oatp2 in TR-BNBs might reflect differences between the environments of CNS and PNS, that is, the former has synapses and the latter does not. Hence, at BNB, as at BBB, many transporters might exist and perform the active influx and efflux transports of required nutrients and potentially harmful substances to peripheral nerves.

We also confirmed the expression of p-glycoprotein and GLUT-1, which are the representative transporters functioning at BBB, and claudin-5, occludin, and claudin-12, which are fundamental $\mathrm{TJ}$ proteins at $\mathrm{BBB}$, in endoneurial microvessels in vivo. Furthermore, our RT-PCR analysis using rat tissues suggests that only the microvascular endothelial cells of the brain and endoneurium have barrier properties, whereas the endothelium in other organs that do not have barrier systems such as the aorta and kidneys do not.

Altogether, we firstly showed direct evidence that PnMECs in BNB have highly specialized characteristics as barrier-forming cells, sharing TJ molecules and various transporters with BBB-forming endothelial cells. Further analyses to elucidate the common features and different aspects between BNB and BBB are needed because findings of such analyses should lead to the development of novel therapies for many autoimmune peripheral nervous diseases.

Acknowledgments. This work was supported in part by a Neuroimmunological Disease Research Committee grant from the Ministry of Health, Labour and Welfare, Japan and also by a Grant-in-Aid for Scientific Research from the Ministry of Education, Science, Sports and Culture, Japan.

\section{References}

Argall, K.G., Armati, P.J., and Pollard, J.D. 1994. A method for the isolation and culture of rat peripheral nerve vascular endothelial cells. Mol. Cell. Neurosci., 5: 413-417.

Asaba, H., Hosoya, K., Takanaga, H., Ohtsuki, S., Tamura, E., Takizawa, T., and Terasaki, T. 2000. Blood-brain barrier is involved in the efflux transport of a neuroactive steroid, dehydroepiandrosterone sulfate, via organic anion transporting polypeptide 2. J. Neurochem., 75: 19071916.

Bazzoni, G., Martinez-Estrada, O.M., Orsenigo, F., Cordenonsi, M., Citi, S., and Dejana, E. 2000. Interaction of junctional adhesion molecule with the tight junction components ZO-1, cingulin, and occludin. J. Biol. Chem., 275: 20520-20526.

Bell, M.A. and Weddell, A.G.M. 1984. A descriptive study of the blood vessels of the sciatic nerve in the rat, man, and other mammals. Brain, 107: 871-898.

Biernacki, K., Prat, A., Blain, M., and Antel, J.P. 2004. Regulation of cellular and molecular trafficking across human brain endothelial cells by Th1- and Th2-polarized lymphocytes. J. Neuropathol. Exp. Neurol., 63: $223-232$.

Boddingius, J. 1984. Ultrastructural and histophysiological studies on the blood-nerve barrier and perineurial barrier in leprosy neuropathy. Acta. Neuropathol., 64: 282-296.

Deli, M.A., Abraham, C.S., Kataoka, Y., and Niwa, M. 2005. Permeability studies on in vitro blood-brain barrier models: physiology, pathology, and pharmacology. Cell. Mol. Neurobiol., 25: 59-127.

Furuse, M., Hirase, T., Itoh, M., Nagafuchi, A., Yonemura, S., Tsukita, S., and Tsukita, S. 1993. Occludin: A novel integral membrane protein 
localizing at tight junctions. J. Cell. Biol., 123: 1777-1788.

Honda, M., Nakagawa, S., Hayashi, K., Kitagawa, N., Tsutsumi, K. Nagata, I., and Niwa, M. 2006. Adrenomedullin Improves the BloodBrain Barrier Function Through the Expression of Claudin-5. Cell. Mol. Neurobiol., 26: 109-118.

Hosoya, K., Takashima, T., Tetsuka, K., Nagura, T., Ohtsuki, S., Takanaga, H., Ueda, M., Yanai, N., Obinata, M., and Terasaki, T. 2000. mRNA expression and transport characterization of conditionally immortalized rat brain capillary endotherial cell lines; a new in vitro BBB model for drug targeting. J. Drug. Target., 8: 357-370.

Hosoya, K., Kondo, T., Tomi, M., Takanaga, H., Ohtsuki, S., and Terasaki, T. 2001. MCT1-mediated transport of L-lactic acid at the inner bloodretinal barrier: a possible route for delivery of monocarboxylic acid drugs to the retina. Pharm. Res., 18: 1669-1676.

Hosoya, K., Tomi, M., Takayama, M., Komokata, Y., Nakai, D., Tokui, T., Nishimura, K., Ueda, M., Obinata, M., Hori, S., Ohtsuki, S., Amidon, G.L., and Terasaki, T. 2004. Transporter mRNA expression in a conditionally immortalized rat small intestine epithelial cell line (TR-SIE). Drug. Metab. Pharmacokinet., 19: 264-269.

Ito, A., Yamaguchi, K., Tomita, H., Suzuki, T., Onogawa, T., Sato, T., Mizutamari, H., Mikkaichi, T., Nishio, T., Suzuki, T., Unno, M., Sasano, H., Abe, T., and Tamai, M. 2003. Distribution of rat organic anion transporting polypeptide-E (oatp-E) in the rat eye. Invest. Ophthalmol. Vis. Sci., 44: 4877-4884.

Iwasaki, T., Kanda, T., and Mizusawa, H. 1999. Effects of pericytes and various cytokines on integrity of endothelial monolayer originated from blood-nerve barrier: an in vitro study. J. Med. Dent. Sci., 46: 31-40.

Jonker, J.W., Merino, G., Musters, S., van Herwaarden, A.E., Bolscher, E., Wagenaar, E., Mesman, E., Dale, T.C., and Schinkel, A.H. 2005. The breast cancer resistance protein BCRP (ABCG2) concentrates drugs and carcinogenic xenotoxins into milk. Nature. Med., 11: 127-129.

Kanai, Y., Segawa, H., Miyamoto, K., Uchino, H., Takeda, E., and Endou, H. 1998. Expression cloning and characterization of a transporter for large neutral amino acids activated by the heavy chain of 4F2 antigen (CD98). J. Biol. Chem., 273: 23629-23632.

Kanda, T., Yoshino, H., Ariga, T., Yamawaki, M., and Yu, R.K. 1994. Glycosphingolipid antigens in cultured microvascular bovine brain endotherial Cells: Sulfoglucuronosyl paragloboside as a target of monoclonal IgM in demyelinative neuropathy. J. Cell. Biol., 126: 235-246.

Kanda, T., Iwasaki, T., Yamawaki, M., and Ikeda, K. 1997. Isolation and culture of bovine endothelial cells of endoneurial origin. J. Neurosci. Res., 49: 769-777.

Kanda, T., Yamawaki, M., Iwasaki, T., and Mizusawa, H. 2000. Glycosphingolipid antibodies and blood-nerve barrier in autoimmune demyelinative neuropathy. Neurology, 54: 1459-1464.

Kanda, T., Numata, Y., and Mizusawa, H. 2004. Chronic inflammatory demyelinating polyneuropathy: decreased claudin- 5 and relocated ZO-1. J. Neurol. Neurosurg. Psychiatry., 75: 765-769.

Kido, Y., Tamai, I., Okamoto, M., Suzuki, F., and Tsuji, A. 2000. Functional clarification of MCT1-mediated transport of monocarboxylic acids at the blood-brain barrier using in vitro cultured cells and in vivo BUI studies. Pharm. Res., 17: 55-62.

Kobayashi, M., Inoue, K., Warabi, E., Minami, T., and Kodama, T. 2005. A simple method of isolating mouse aortic endothelial cells. $J$. Atheroscler. Thromb., 12: 138-142.

Lach, B., Rippstein, P., Atack, D., Afar, D.E., and Gregor, A. 1993. Immunoelectron microscopic localization of monoclonal $\operatorname{IgM}$ antibodies in gammopathy associated with peripheral demyelinative neuropathy. Acta. Neuropathol., 85: 298-307.

Lai, C.H., Kuo, K.H., and Leo, J.M. 2005. Critical role of actin in modulating BBB permeability. Brain Res. Brain Res. Rev., 50: 7-13.

Latker, C.H., Wadhwani, K.C., Balbo, A., and Rapoport, S.I. 1991. Blood- nerve barrier in the frog during Wallerian degeneration: Are axons necessary for maintenance of barrier functions? J. Comp. Neurol., 309: 650-664.

Mori, S., Takanaga, H., Ohtsuki, S., Deguchi, T., Kang, Y.S., Hosoya, K., and Terasaki, T. 2003. Rat organic anion transporter 3 (rOAT3) is responsible for brain-to-blood efflux of homovanillic acid at the abluminal membrane of brain capillary endothelial cells. J. Cereb. Blood. Flow. Metab., 23: 432-440.

Nitta, T., Hata, M., Gotoh, S., Seo, Y., Sasaki, H., Hashimoto, N., Furuse, M., and Tsukita, S. 2003. Size-selective loosening of the blood-brain barrier in claudin-5-deficient mice. J. Cell. Biol., 161: 653-660.

Ohtsuki, S., Tachikawa, M., Takanaga, H., Shimizu, H., Watanabe, M., Hosoya, K., and Terasaki, T. 2002. The blood-brain barrier creatine transporter is a major pathway for supplying creatine to the brain. $J$. Cereb. Blood. Flow. Metab., 22: 1327-1335.

Ohtsuki, S., Tomi, M., Hata, T., Nagai, Y., Hori, S., Mori, S., Hosoya, K., and Terasaki, T. 2005. Dominant expression of androgen receptors and their functional regulation of organic anion transporter 3 in rat brain capillary endothelial cells; comparison of gene expression between the blood-brain and -retinal barriers. J. Cell. Physiol., 204: 896-900.

Ohtsuki, S., Sato, S., Yamaguchi, H., Kamoi, M., Asashima, T., and Terasaki, T. 2007. Exogenous expression of claudin-5 induces barrier properties in cultured rat brain capillary endothelial cells. J. Cell. Physiol., 210: 81-86.

Pardridge, W.M. 2005. The blood-brain barrier: bottleneck in brain drug development. NeuroRx, 2: 3-14.

Poduslo, J.F., Curran, G.L., and Berg, C.T. 1994. Macromolecular permeability across the blood-nerve and blood-brain barriers. Proc. Natl. Acad. Sci. U.S.A., 91: 5705-5709.

Rechthand, E., Smith, Q.R., and Rapoport, S.I. 1985. Facilitated transport of glucose from blood into peripheral nerve. J. Neurochem., 45: 957964.

Saito, T., Zhang, Z.J., Shibamori, Y., Ohtsubo, T., Noda, I., Yamamoto, T., and Saito, H. 1997. P-glycoprotein expression in capillary endothelial cells of the 7 th and 8 th nerves of guinea pig in relation to blood-nerve barrier sites. Neurosci. Lett., 232: 41-44.

Shi, L.Z. and Zheng, W. 2005. Establishment of an in vitro brain barrier epithelial transport system for pharmacological and toxicological study. Brain Res., 1057: 37-48.

Soderfeldt, B. 1974. The perineurium as a diffusion barrier to protein tracers. Influence of histamine, serotonine and bradykinine. Acta. Neuropathol., 27: 55-60.

Takahashi, R., Hirabayashi, M., Yanai, N., Obinata, M., and Ueda, M. 1999. Establishment of SV40-tsA58 transgenic rats as a source of conditionally immortalized cell lines. Exp. Anim., 48: 255-261.

Terasaki, T., Ohtsuki, S., Hori, S., Takanaga, H., Nakashima, E., and Hosoya, K. 2003. New approaches to in vitro models of blood-brain barrier drug transport. Drug. Discov. Today., 8: 944-954.

Tomi, M. and Hosoya, K. 2004. Application of magnetically isolated rat retinal vascular endothelial cells for the determination of transporter gene expression levels at the inner blood-retinal barrier. J. Neurochem., 91: 1244-1248.

Wadhwani, K.C., Smith, Q.R., and Rapoport, S.I. 1990. Facilitated transport of L-phenylalanine across blood-nerve barrier of rat peripheral nerve. Am. J. Physiol., 258: 1436-1444.

Weerasuriya, A. and Rapoport, S.I. 1986. Endoneurial capillary permeability to $\left[{ }^{14} \mathrm{C}\right]$ sucrose in frog sciatic nerve. Brain Res., 375: 150-156.

Weerasuriya, A., Curran, G.L., and Poduslo, J.F. 1989. Blood-nerve transfer of albumin and its implications for the endoneurial microenvironment. Brain Res., 494: 114-121.

(Received for publication, May 9, 2007 and accepted, November 3, 2007) 